\title{
Experimental estimation of the luminosity of the Sun
}

\author{
Salvador $\mathrm{Gil}^{\mathrm{a})}$ \\ Escuela de Ciencia y Tecnología, Universidad Nacional de San Martín, Provincia de Buenos Aires, \\ Argentina 1653 \\ and Departamento de Física "J. J. Giambiagi”- FCEyN- Universidad de Buenos Aires, Buenos Aires, \\ Argentina 1428 \\ Mariano Mayochi \\ Departamento de Física “J. J. Giambiagi”- FCEyN- Universidad de Buenos Aires, Buenos Aires, \\ Argentina 1428 \\ Leonardo J. Pellizza \\ Departamento de Física "J. J. Giambiagi"- FCEyN- Universidad de Buenos Aires, Buenos Aires, \\ Argentina 1428 \\ and Instituto de Astronomía y Física del Espacio (CONICET/UBA), Argentina 1428
}

(Received 10 October 2005; accepted 10 March 2006)

\begin{abstract}
We determine the solar constant experimentally using two independent techniques that allow us to study the dependence of the solar irradiation on the zenith angle and the characteristics of the light extinction in the atmosphere. Our result for the solar luminosity agrees within $7 \%$ of the accepted value. This value can be used to estimate the luminosity or power radiated by the Sun. The experiment is inexpensive and conceptually easy to perform and understand. (C) 2006 American Association of Physics Teachers.
\end{abstract}

[DOI: 10.1119/1.2192789]

\section{INTRODUCTION}

A detailed understanding of how the Sun produces its energy is crucial for developing models of more distant stars. In 1920 Arthur Eddington suggested that nuclear fusion powered the Sun, but efforts to confirm this hypothesis have encountered important difficulties. At the core of this problem is the challenge to reconcile the total power produced by the Sun and the number of nuclear reactions that take place in its interior.

Each nuclear fusion reaction produces a well-defined amount of energy and neutrinos. ${ }^{1-3}$ Because the interaction of neutrinos with matter is so weak, the neutrinos produced in the core of the Sun can easily reach the Earth. The number of neutrinos per unit time generated by the Sun (its neutrino luminosity) can be directly measured and the rate of nuclear reactions inferred. The solar envelope is opaque to electromagnetic radiation. Because the Sun is in steady state, the power generated by the solar core balances the electromagnetic radiation emitted by the solar photosphere (its luminosity). The radiation generated in the photosphere can be measured on the Earth, giving a second, indirect way to estimate the solar nuclear reaction rate. Hence, if the nuclear fusion model for the solar energy production is correct, the rate inferred by both ways should be equal.

In the late 1960s the first solar neutrino observations provided evidence for a discrepancy between the two measurements. The nuclear reaction rate inferred from the neutrino luminosity was about one-third of that expected from the electromagnetic luminosity. This discrepancy gave birth to a question whose answer eluded physicists for three decades. Recent results from the Sudbury Neutrino Observatory $(\mathrm{SNO})^{1,2,4,5}$ have given a satisfactory explanation for the deficit of neutrinos that earlier experiments had found.

Understanding the way the Sun produces its energy is also related to the possible long-term variation of the solar luminosity and its connection to current questions about the origin of global warming. It is important to establish whether the causes are natural, anthropogenic, or both, a point that is still unclear. ${ }^{6}$ Several experiments are under way to help to elucidate this question. ${ }^{7}$ Finally, the solar irradiance on Earth, the amount of sunlight that reaches the Earth surface, is an important source of energy. The study of its characteristics is useful for many practical applications.

There are relatively few ways to measure the solar luminosity that are possible for undergraduate students. ${ }^{8}$ In this article we present a simple and low cost experiment to measure the solar luminosity. The method is based on measuring the solar irradiance at the surface of the Earth and light extinction in the Earth's atmosphere. By combining both results we obtain the solar constant, the flux of solar radiation at the Earth's orbit outside the atmosphere, which is directly related to the solar luminosity. In this way the value of the solar luminosity can be obtained with an accuracy of about $7 \%$.

The measurement of the solar irradiance has received relatively more attention in the past, mostly because of the practical uses of solar energy. ${ }^{9-13}$ The measurement techniques vary in sophistication from very simple and suitable to do at home $^{9}$ to more elaborate and precise ones. ${ }^{8,10-12}$ An interesting approach consists of using two identical blackened surfaces, one of which is shielded from the Sun. The measurement of the electrical power needed to bring the shaded sample to the same temperature as the exposed one gives an estimation of the solar irradiance ${ }^{10,11,13}$

In this paper we present a simple alternative to this technique that allows us to obtain an absolute estimation of the solar irradiation with reasonable accuracy. It can be carried out in a teaching laboratory and the relevant physics of the problem emerges clearly.

\section{THEORETICAL CONSIDERATIONS}

\section{A. Heating a plate exposed to the Sun}

Consider a rectangular metal plate of area $A$ and thickness $t_{p}$ exposed to the Sun in such a way that its surface is perpendicular to the incident solar radiation. We let $I_{\text {gnd }}$ be the 
solar irradiance, the total intensity of solar energy at ground level. We denote by $r$ the effective reflectivity of the face of the plate exposed to the Sun. If the plate is prepared to minimize heat conduction and if its temperature does not differ appreciably from that of the surrounding media, then the main mechanism of heat dissipation will be convection. ${ }^{14}$ In this case, Newton's law of cooling can be used to model this energy dissipation, ${ }^{15}$

$$
\left\lfloor\frac{d Q}{d t}\right\rfloor_{\text {convection }}=m_{p} c_{p} \frac{d T}{d t}=-k m_{p} c_{p}\left(T-T_{0}\right) .
$$

Here $T$ represents the temperature of the plate, $T_{0}$ the temperature of the surroundings, $m_{p}$ and $c$ are the mass and specific heat of the plate, respectively, $k$ is the heat transfer constant of the plate, $t$ is the time, and $d Q / d t$ is the rate of energy loss. When the plate is exposed to the Sun, we have

$$
\frac{d Q}{d t}=m_{p} c \frac{d T}{d t}=-k m_{p} c\left(T-T_{0}\right)+I_{\mathrm{gnd}} \varepsilon A,
$$

where $\varepsilon=1-r$ is the effective emissivity of the face of the plate exposed to solar radiation. Equation (2) can be written as:

$$
\frac{d T}{d t}+k T=k T_{0}+\frac{A}{m_{p} c} \varepsilon I_{\mathrm{gnd}},
$$

and because

$$
m_{p}=\rho_{p} A t_{p},
$$

where $\rho_{p}$ is the density of the plate, we can rearrange Eq. (3) to obtain

$$
\frac{d T}{d t}+k T=k T_{0}+\frac{1}{\rho_{p} c t_{p}} \varepsilon I_{\mathrm{gnd}} .
$$

Equation (5) can be expressed as

$$
\frac{d T}{d t}+k T=k T_{\infty}
$$

where

$$
T_{\infty}=\frac{1}{\rho_{p} c t_{p}} \frac{\varepsilon I_{\mathrm{gnd}}}{k}+T_{0}
$$

represents the equilibrium temperature of the plate if it is exposed indefinitely to the Sun.

The solution of the differential equation (6) with the initial condition $T(0)=T_{i}$ is

$$
T(t)=\left[\left(T_{i}-T_{\infty}\right)\right] \cdot \exp (-k t)+T_{\infty} .
$$

Because $T_{\infty}>T_{0}$, if the plate is initially cooler than the ambient temperature $\left(T_{0}>T_{i}\right)$, then for some finite $t_{0}>0, T$ $=T_{0}$, and from Eq. (2) or (5), we obtain

$$
\left.\frac{d T(t)}{d t}\right|_{t=t_{0}}=\frac{\varepsilon}{\rho_{p} c} \frac{I_{\mathrm{gnd}}}{t_{p}} .
$$

Hence, the slope of the curve $T(t)$ when the temperature of the plate is equal to the ambient temperature $T_{0}$ is proportional to the solar irradiance $I_{\text {gnd }}$ and inversely proportional to the thickness of the plate. Equation (9) can be used to obtain an estimate of the solar constant, independent of the heat transfer coefficient $k$. By analyzing the energy balance in Eq. (2), we see that Eq. (9) will hold even if we

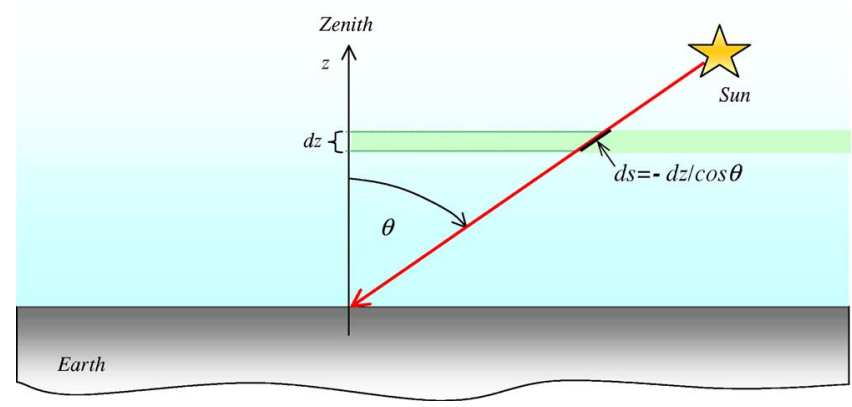

Fig. 1. The plane-parallel model for the local atmosphere; $\theta$ is the angle between the zenith and the direction of the Sun and $d s$ is the differential path traveled by solar light between height $z$ and $z+d z$.

take into account radiation or other types of energy transfer mechanisms. The physical reason is that when the temperature of the plate equals the temperature of the surroundings, the net energy flux between the plate and its environment is zero, and the variation of the plate temperature is due solely to the influx of energy from the Sun. In this regard Eq. (9) is a more robust result than Eq. (7), because the asymptotic temperature $T_{\infty}$ depends on the detailed energy dissipation mechanism, whereas Eq. (9) does not.

The effective heat transfer constant $k$ of the plate can be measured by shielding plates from the Sun and measuring how the plates cool once they have been heated to some temperature $T_{h}>T_{0}$. For plates exposed to the Sun and temperatures close to the ambient temperature, we can write according to Eq. (3),

$$
\left.\frac{d T}{d t}\right|_{T \approx T_{0}}=\frac{\varepsilon}{\rho_{p} c} \frac{I_{\mathrm{gnd}}}{t_{p}}-k\left(T-T_{0}\right) .
$$

The last term on the right-hand side of Eq. (10) at $T \approx T_{0}$ does not affect $d T / d t$, but causes the second derivative of the function $T(t)$ to be negative.

\section{B. Light extinction in the Earth's atmosphere}

To obtain an estimate of the luminosity of the Sun by measuring the flux at the surface of the Earth, it is necessary to understand how solar light is absorbed as it travels through the Earth's atmosphere. Almost all of the absorption occurs in the lowest, most dense layer of the atmosphere (the troposphere), which is only about $10 \mathrm{~km}$ thick. The radius of curvature of the troposphere is the Earth radius, $6371 \mathrm{~km}$, which is almost $10^{3}$ times larger than its thickness. It is sufficiently accurate for our purposes to consider the solar light attenuation through a local plane layer of atmosphere (see Fig. 1). Moreover, the spherical symmetry of the atmosphere allows us to consider the properties of the atmosphere as only a function of height above the Earth's surface, independently of the azimuth angle. The plane atmosphere approximation is not valid when solar light comes from points very near the horizon, because this light travels a longer path through the atmosphere rendering invalid any local approximation. Comparison to more accurate spherical models shows that the plane-parallel approximation can be used up to zenith angles of $\sim 75^{\circ}$. Hence our discussion will be limited to these regions of the sky, which is large enough for our purpose. The Appendix presents a more elaborate model. 
As we have discussed we assume that the air density varies only with the vertical coordinate $z$. Air is a mixture of different gases. The constants $\sigma_{T}^{(i)}$ and $n^{(i)}(z)$ represent the extinction (scattering plus absorption) cross section and the number of moles per unit of volume of component $i$ of the air at height $z$ above the Earth's surface. According to the Beer-Lambert law, ${ }^{16}$

$$
d I=-\left(\sum_{i} \sigma_{T}^{(i)} n^{(i)}(z) N_{A} d s\right) I(z)=-K(z) I(z) d z / \cos \theta,
$$

where $N_{A}$ is Avogadro's number and $d I$ represents the change of intensity of a pencil of light from the Sun as it traverses the length of air $d s$. We denote by $\theta$ the zenith angle of the Sun. From the geometry of the system (see Fig. $1), d s=\mu d z$, where $\mu=1 / \cos \theta$. We define the extinction coefficient as $K(z)=\sum_{i} \sigma_{T}^{(i)} n^{(i)}(z) N_{A}$. Equation (11) can be integrated between $z=0$ and infinity to give

$$
I(z=0)=I_{\text {gnd }}=I_{0} \exp \left(-\mu K_{\text {eff }}\right),
$$

where

$$
K_{\text {eff }}=\int_{0}^{\infty} K(z) d z=N_{A} \sum_{i} \sigma_{T}^{(i)}\left(\int_{0}^{\infty} n^{(i)}(z) d z\right) .
$$

Equation (12) relates the Solar constant $I_{0}$ to the solar irradiation at ground level. Measuring the solar irradiation at ground level, $I_{\text {gnd }}$, as a function of the zenith angle $\theta$ allows us to obtain $I_{0}$ and $K_{\text {eff }}$ and avoids the need for an independent determination of the latter.

\section{THE EXPERIMENT}

We used two independent but complementary setups to measure the solar constant. Our first setup consists of aluminum plates $15 \mathrm{~cm} \times 15 \mathrm{~cm}$ in size and thickness $t_{p}=2,2.6$, and $3 \mathrm{~mm}$. A NTC thermistor (resistance at $25{ }^{\circ} \mathrm{C}=5 \mathrm{k} \Omega$ ) was attached to the back side of each plate at its center with silicon grease to improve their thermal contact. The thermistors were connected in series to a $10 \mathrm{k} \Omega$ resistor and a power supply of $5 \mathrm{~V}$. The voltage from the thermistor was monitored by a data acquisition system ${ }^{17}$ connected to a personal computer. The thermistors were calibrated so that the temperature could be recorded. We could record the temperatures of the three plates at the same time. To prevent gusts of air that would alter the temperature of the plates, we built a box of expanded polyurethane $20 \mathrm{~cm}$ high, $60 \mathrm{~cm}$ wide, and $60 \mathrm{~cm}$ long with an open front so that the solar radiation could reach the plates that were located in its interior. A schematic diagram of this setup is shown in Fig. 2. A $3 \mathrm{~cm}$ slot allowed the plates to be placed inside and simultaneously achieve easy thermalization of the interior of the box with the surrounding air. A conventional mercury thermometer located close to the plates was used to monitor the ambient temperature. Up to three plates could be placed in the box for evaluation. When the shadow of the walls on the back face of the box vanished, it indicated that the plates were perpendicular to the solar radiation.

The aluminum plates were painted using spray lacquer flat black to reduce their reflectance. To characterize the reflectance of the painted surface, we used an integrating sphere attached to a Beckman UV5270 infrared-visible spectrophotometer. We obtained a reflectivity $r=5.0 \pm 0.5 \%$ for the

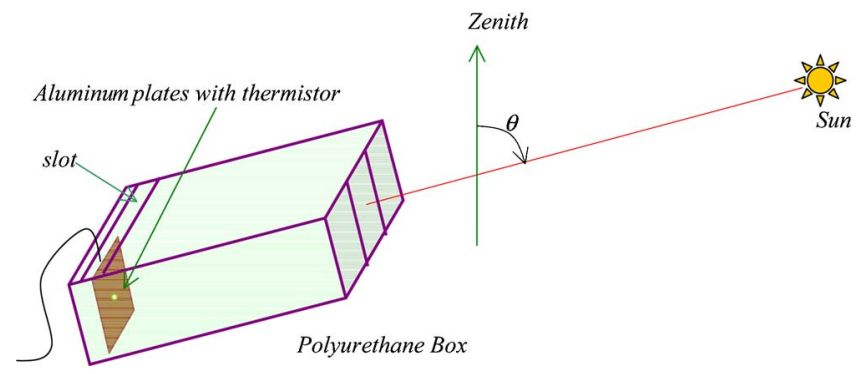

Fig. 2. Experimental setup, showing the expanded polyurethane box housing the aluminum plates conected to a thermistor on their back.

plates, in the range of $300-1800 \mathrm{~nm}$. The total effective reflectivity of the plates, weighted by the solar spectrum, resulted in $r=5.4 \pm 0.3 \%$.

Before each measurement the plates were cooled using dry ice to a temperature of about $10{ }^{\circ} \mathrm{C}$ below the ambient temperature. The temperatures of the plates were measured at $1 \mathrm{~s}$ intervals after the dry ice was removed to construct a temperature versus time curve. We did our measurements on clear days with no clouds in sight. We also avoided windy days. Small pieces of yarn were used to visually monitor the state of the air. We registered the local time of each measurement, which along with the geographical coordinates allowed us to obtain the zenith angle of the Sun. ${ }^{18}$

We also used a second experimental technique based on using a photovoltaic solar cell as a detector, which directly measures the solar irradiance. It consisted of a small piece of commercial grade solar cell ${ }^{19}$ with an aperture of about $10 \mathrm{~mm}^{2}$. The detector was located inside a blackened tube that prevents scattered light from the sky from reaching the detector. By using the shadow of the tube as a reference, the detector was positioned before each measurement so that it was perpendicular to the incident solar radiation. The voltage in this device was measured with a digital voltmeter and is proportional to the solar irradiation. ${ }^{8,12}$ This type of detector is sensitive mainly to the visible and near IR radiation. ${ }^{8,12}$ To obtain an absolute measurement of the solar irradiation, we compared our results to Eppley Laboratory Pyrheliometer at the San Miguel Observatory of Buenos Aires. The detector gives a very simple and quick measurement of the solar irradiation and is very adequate for studying the variation of irradiation with the zenith angle of the Sun; however, a calibration against a reference pyrheliometer is essential.

\section{RESULTS AND DISCUSSION}

We first assess the validity of the hypothesis in Eq. (1), namely, that the heat loss in the plates for temperatures that are not very different from the ambient temperature is proportional to $T-T_{0}$. We warmed the plates using a heat gun. Then we placed them inside the expanded polyurethane box in the same manner as would be used in the actual measurements, but shielded from the Sun. We measured their temperature as a function of time, which allowed us to compute $d T / d t$ by numerical differentiation of the data. In Fig. 3 we show the variation of $d T / d t$ as a function of $T-T_{0}$ for two of the plates, together with linear fits to the data. Figure 3 shows that the rate of change of the temperature of the plates is proportional to $T-T_{0}$.

Next, we cooled the plates using dry ice to about $15{ }^{\circ} \mathrm{C}$ below the ambient temperature $T_{0}$. Then we mounted the 


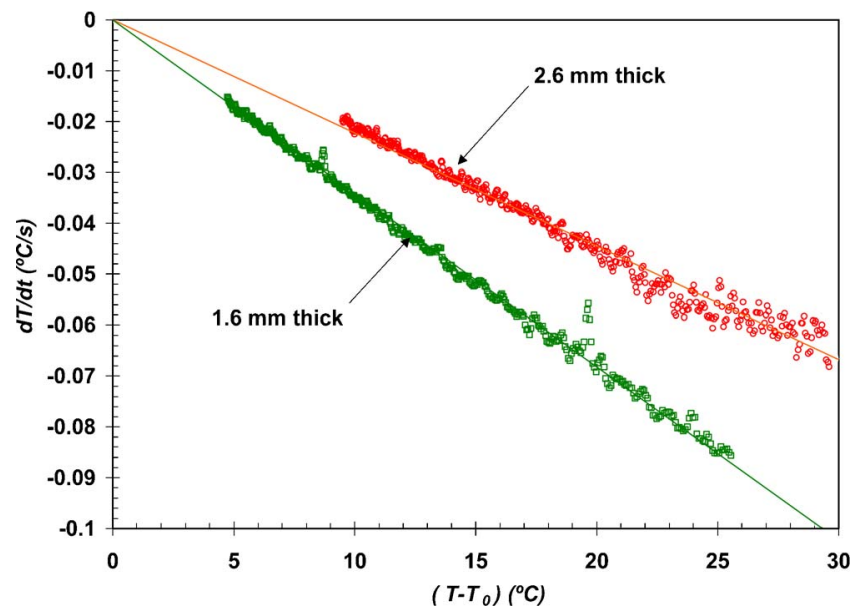

Fig. 3. The variation of $d T / d t$ for two unexposed plates, previously heated by a heat gun, as a function of the temperature difference $T-T_{0}$. The ambient temperature was $T_{0}=22{ }^{\circ} \mathrm{C}$. The thickness of the plates was 2.6 and $1.6 \mathrm{~mm}$, respectively. The continuous lines are linear fits to the data. These results indicate that our plates follow Newton's law of cooling. The slope of these lines allows us to estimate the heat transfer constant $k$ of the plates.

plates inside the expanded polyurethane box. They were placed perpendicularly to the solar rays and their temperature was measured. It took about $2 \mathrm{~min}$ for the plates to reach $T_{0}$ when they were exposed to the Sun. In Fig. 4 we show the variation of the measured temperature of the plates exposed to the Sun as a function of time. For temperatures close to the ambient temperature (within approximately $5{ }^{\circ} \mathrm{C}$ ), a straight line closely fits the data. The slopes of these fits were used to obtain $d T / d t$ at ambient temperature. According to Eq. (9), these results allow us to estimate the solar irradiance $I_{\text {gnd }}$ using tabulated values of the density $\rho_{p}$ and specific heat $c$ of aluminum, and our previously measured values of the reflectivity $r$ and thickness $t_{p}$ of each plate.

Our measurements were performed for different zenith angles $\theta$ of the Sun, which were calculated from the time and date of the measurements and from the latitude of our site. It took us about 20 min to perform the measurement of $I_{\mathrm{gnd}}$ for each angle using our first setup. In Fig. 5 we present the results of the solar irradiation $I_{\text {gnd }}(\theta)$ as a function of $\mu$

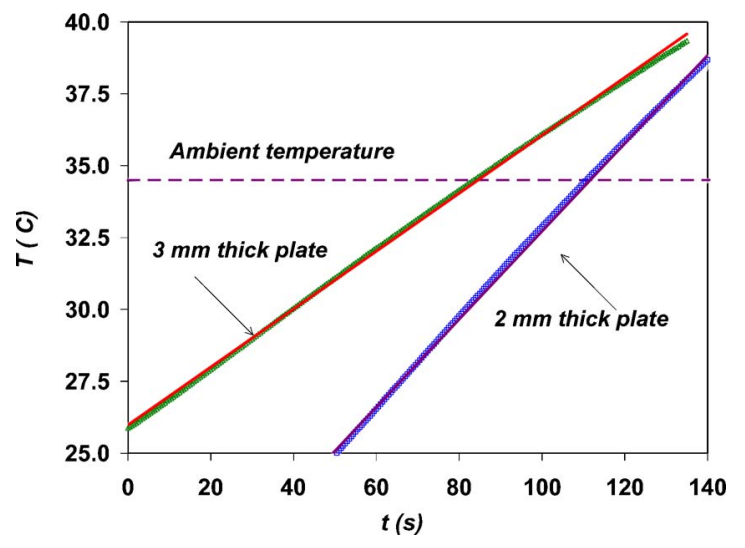

Fig. 4. The variation of the temperature of two plates, with thickness 2 and $3 \mathrm{~mm}$, respectively, exposed to the Sun. The ambient temperature was $34.5^{\circ} \mathrm{C}$. The continuous lines are linear fits to the data. This measurement was carried out in Buenos Aires on 25 September 2004 at 2:15 pm. These data are consistent with a solar irradiation of $755 \mathrm{~W} / \mathrm{m}^{2}$.

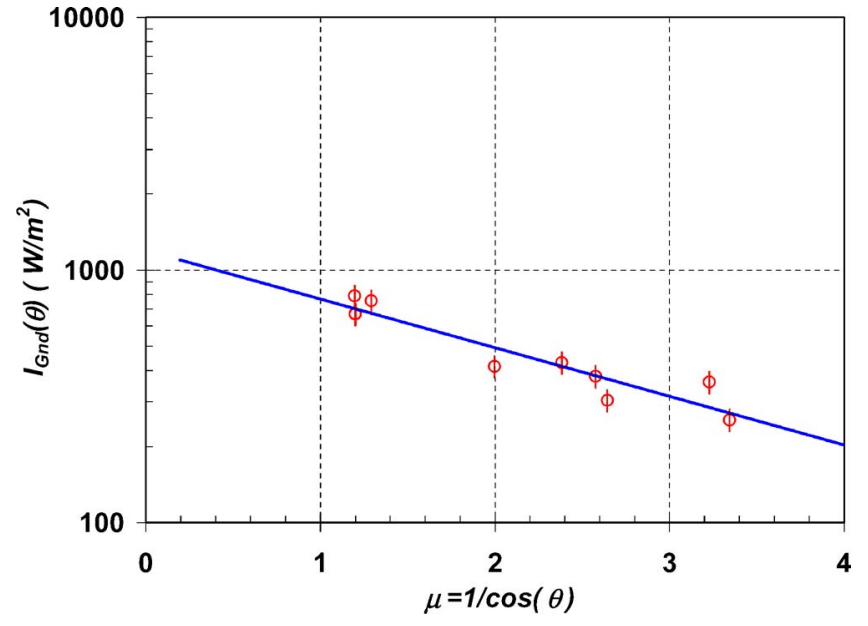

Fig. 5. Experimental results for $I_{\text {gnd }}(\theta)$ as a function of $\mu$, using the heated aluminum plates technique. The continuous line is an exponential fit to the data using Eq. (14). If we extrapolate to $\mu=1 / \cos (\theta) \rightarrow 0$, we obtain the value of the solar constant. $I_{0}=1340 \pm 130 \mathrm{~W} / \mathrm{m}^{2}$.

$=1 / \cos (\theta)$. The trend observed in Fig. 5 suggests that the dependence of the plotted variables can be described by Eq. (12). By a simple extrapolation to $\mu=0$, the value of the solar constant $I_{0}=1340 \pm 130 \mathrm{~W} / \mathrm{m}^{2}$ was obtained from this set of measurements.

We also performed the experiment using the technique based on the solar cell detector. After calibrating the detector we exposed it perpendicularly to the solar rays. We took several measurements of $I_{\mathrm{gnd}}$ at different zenith angles of the Sun, calculated in the same way as before. In Fig. 6 we present the results of the measurement of $I_{\mathrm{gnd}}(\theta)$ as a function of $\mu=1 / \cos (\theta)$. The trend described by Eq. (12) is again well reproduced. The data obtained using the solar cell detector have less dispersion than that obtained using the first setup 1. The results of using the solar cell detector show a systematic deviation from the naive model described by Eq.

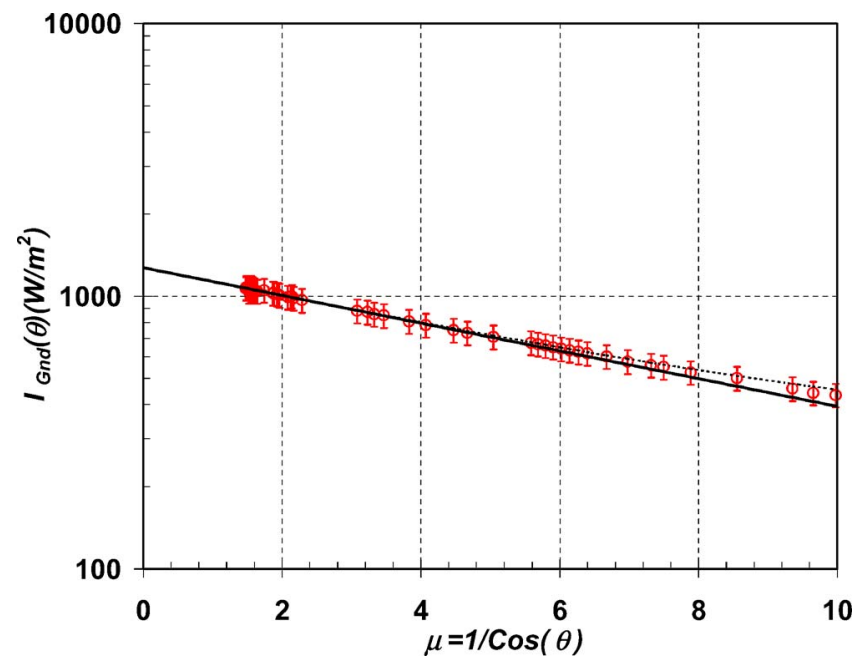

Fig. 6. Measurement of $I_{\text {gnd }}(\theta)$ as a function of $\mu=1 / \cos (\theta)$ using the calibrated solar cell detector. The solid line is an exponential fit to the data. The dashed line is the prediction taking into account the curvature of the Earth as discussed in the Appendix. The value of the solar constant obtained using this technique yields $I_{0}=1270 \pm 110 \mathrm{~W} / \mathrm{m}^{2}$. The measurement was done on 23 April 2005. 
(12) at large zenith angles. To reproduce the results of irradiance at large zenith angles, the effect of the shape of the Earth has to be considered as discussed in the Appendix. The value of the solar constant obtained using the solar cell detector by fitting the model of the Appendix to the data and extrapolating to $\mu=0$ is $I_{0}=1270 \pm 110 \mathrm{~W} / \mathrm{m}^{2}$.

If we use the measured values of the solar constant and the corresponding Sun-Earth distance $d_{\text {Sun-Earth }}$ for the date on which the measurements were made, we would obtain the solar luminosity as

$$
L=4 \pi d_{\text {Sun-Earth }}^{2} I_{0} .
$$

The use of the value of $d_{\text {Sun-Earth }}$ at the date of the measurement is important because the eccentricity of the Earth orbit produces a variation in the Sun-Earth distance of about $\pm 1.7 \%{ }^{8}$ We included this effect in our calculations and combined the results and errors associated with the two independent measurements of the same quantity to obtain a weighted average value of the luminosity of the Sun, $L=(3.57 \pm 0.25)$ $\times 10^{26} \mathrm{~W}$. This result is consistent to within $1.1 \sigma$ of the accepted value, $L=(3.842 \pm 0.015) \times 10^{26} \mathrm{~W} .^{6,20}$

\section{ACKNOWLEDGMENTS}

We would like to acknowledge Dr. C. Bolzi and H. Grossi Gallegos for their assistance in the fabrication and calibration of the photo voltaic cell detector. We also thank Professor C. Waltham for the spectrophotometric measurement of the reflectivities of the plates and Dr. A. Schwint for reading the manuscript. We dedicate this work to two great physicists who have recently passed away and have greatly contributed to our understanding of how the Sun and the stars produce their energy: Hans Bethe and John Bahcall.

\section{APPENDIX: LIGHT EXTINCTION IN THE EARTH'S ATMOSPHERE INCLUDING THE CURVATURE OF THE EARTH}

A more accurate model for light extinction in the atmosphere can be obtained by including effects associated with its curvature. A schematic diagram is shown in Fig. 7, where $R$ represents the Earth radius, $\theta$ is the zenith angle of the Sun, and $z$ is the vertical coordinate of the layer of atmosphere of interest. We take the origin of our coordinate system to be at the point of observation. From the geometry, the equation of the circle that represents the surface of the Earth is

$$
x^{2}+(y+R)^{2}=R^{2},
$$

and the corresponding equation for the layer of atmosphere of height $z$ is

$$
x^{2}+(y+R)^{2}=(R+z)^{2} .
$$

The distance $s$ between the origin and point $P$ is

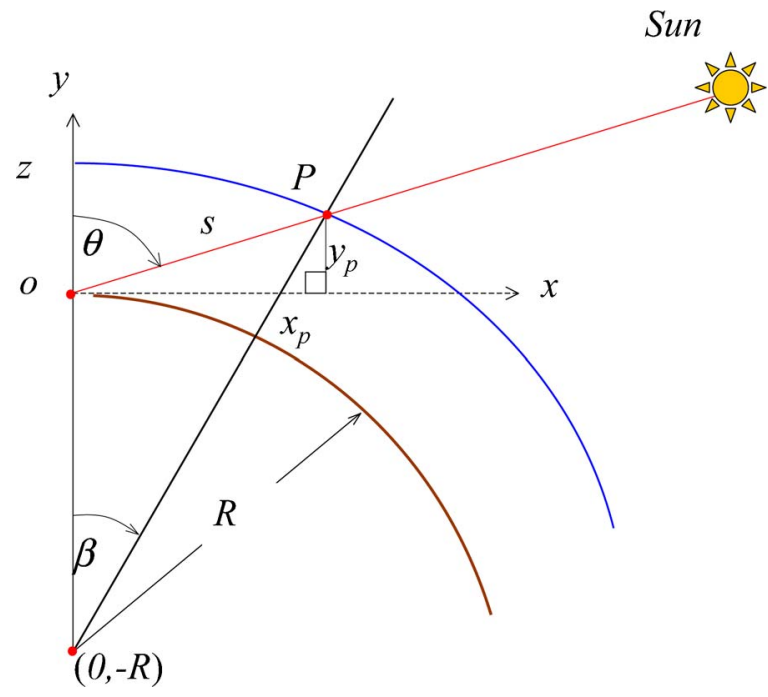

Fig. 7. The Sun at the zenith angle $\theta . R$ is the radius of the Earth. The origin of coordinates is the point of observation $O . z$ is the vertical coordinate of the layer of atmosphere considered.

$$
s^{2}=x_{p}^{2}+y_{p}^{2}=z^{2}+2 R z-2 R y_{p} .
$$

We combine Eq. (A3) with $y_{p}=s \cos \theta$ and obtain

$$
s=R \cos \theta\left\lfloor\sqrt{1+z^{2} / R^{2} \cos ^{2} \theta+2 z / R \cos ^{2} \theta}-1\right\rfloor,
$$

and

$$
d s=\frac{d z}{\cos \theta}\left[\frac{1+z / R}{\sqrt{1+z^{2} / R^{2} \cos ^{2} \theta+2 z / R \cos ^{2} \theta}}\right] .
$$

Note that for $z \ll R, \mathrm{~d} s \rightarrow d z / \cos \theta$, in agreement with the expression used in Eq. (11) in the plane Earth approximation (Fig. 1).

According to the Beer-Lambert law,

$$
\begin{aligned}
\frac{d I}{I(z)}= & -\left(\sum_{i} \sigma_{T}^{(i)} n^{(i)}(z) N_{A} d s\right) \\
= & -\left(\sum_{i} \sigma_{T}^{(i)} n^{(i)}(0) N_{A}\right) \frac{\rho(z)}{\rho_{0}} \frac{d z}{\cos \theta} \\
& \times\left[\frac{1+z / R}{\sqrt{1+z^{2} / R^{2} \cos ^{2} \theta+2 z / R \cos ^{2} \theta}}\right],
\end{aligned}
$$

where the density of the atmosphere at height $z$ is given by $\rho(z)$. Here we assume that $n^{(i)}(z)=n^{(i)}(0) \rho(z) / \rho_{0}$, where $\rho_{0}$ is the density of the atmosphere at ground level. As was indicated in Sec. II, the superindex $i$ represents the different components that constitute the Earth atmosphere. If we define the extinction coefficient $K=\Sigma_{i} \sigma_{T}^{(i)} n^{(i)}(0) N_{A}$ and assume an isothermal atmosphere, $\rho(z)=\rho_{0} \exp (-z / h)$, Eq. (A6) can be integrated between $z=0$ and infinity to give

$$
I(z=0)=I_{\text {gnd }}=I_{0} \exp \left\{-K \int_{z=0}^{\infty}\left[\frac{(1+z / R) e^{-z / h}}{\sqrt{1+z^{2} / R^{2} \cos ^{2} \theta+2 z / R \cos ^{2} \theta}} \frac{d z}{\cos \theta}\right]\right\} .
$$

Equation (A7) relates the solar constant $I_{0}$ to the solar irradiation at ground level $I(z=0)=I_{\text {gnd. }}$. The constant $h=8429 \mathrm{~m}^{21,22}$

Equation (A7) cannot be expressed analytically in closed form, but can be integrated numerically. The assumption of an isothermal atmosphere at low altitude, where the light absorption is more important, is reasonable and simplifies the calcula- 
tion. A more realistic model of the atmosphere, such as an adiabatic atmosphere, does not alter the results appreciably. Because most of the contributions to the integral in Eq. (A7) occur for $z \ll R$ and $\theta<90^{\circ}$, Eq. (A7) can be simplified to

$$
\begin{aligned}
I(z=0) & \approx I_{0} \exp \left\{-K \int_{z=0}^{\infty}\left[\frac{e^{-z / h}}{\sqrt{1+2 z / R \cos ^{2} \theta}} \frac{d z}{\cos \theta}\right]\right\} \approx \sqrt{\frac{\pi R}{2 h}} \exp \left(\frac{R \cos ^{2} \theta}{2 h}\right) \operatorname{erfc}\left(\sqrt{\frac{R \cos ^{2} \theta}{2 h}}\right) \\
& \approx I_{0} \exp \left\{-\frac{K h}{\cos \theta}\left[1-\frac{h}{R} \sec ^{2} \theta\right]\right\} \approx I_{0} \exp \left\{-\frac{K h}{\cos \theta}\left[1-0.0012 \sec ^{2} \theta\right]\right\} .
\end{aligned}
$$

Here $\operatorname{erfc}(x)$ is the complementary error function. The effect of refraction in the atmosphere can be included in Eq. (A8) by taking $R=(7 / 6) R_{\text {Earth. }}{ }^{23}$

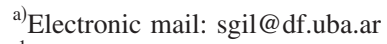

${ }^{1}$ Arthur B. McDonald, Joshua R. Klein, and David L. Wark, "Solving the solar neutrino problem," Sci. Am. 288(4), 22-30 (2003).

${ }^{2}$ J. N. Bahcall, "How the Sun shines," J. R. Astron. Soc. Can. 94(6), 219-227 (2000), 〈http://www.sns.ias.edu/ jnb/>, and $\langle$ http://nobelprize.org/physics/articles $\rangle$.

${ }^{3}$ W. C. Haxton and B. R. Holstein, "Neutrino physics: An update," Am. J. Phys. 72(1) 18-24 (2004).

${ }^{4}$ C. Waltham, "Teaching neutrino oscillations," Am. J. Phys. 72(6), 742752 (2004).

${ }^{5}$ The results of SNO have helped to answer the long-standing question of why the Sun and the stars shine (Refs. 1 and 2) and provided evidence for neutrinos undergoing a flavor change on their trip to the Earth (Refs. 3 and 4). In 2002 R. Davis and M. Koshiba, pioneers in experimental neutrino astrophysics, shared the Nobel Prize in physics with R. Giacconi for their contribution to solving this puzzle (Refs. 1 and 3).

${ }^{6} \mathrm{C}$. Fröhlich and J. Lean, "Solar radiative output and its variability: Evidence and mechanisms," Astron. Astrophys. Rev. 12(4), 273-320 (2004).

${ }^{7}$ See $\langle$ http://science.nasa.gov/headlines/y2003/17jan_solcon.htm〉.

${ }^{8}$ D. L. DuPuy, "Measuring solar luminosity with a photodiode," Am. J. Phys. 57(9), 826-828 (1989).

${ }^{9}$ B. G. Eaton, R. DeGeer, and P. Freier, "The solar constant: A take home lab," Phys. Teach. 42, 51-52 (2004). Original published in Phys. Teach. 15, 172-173 (1977).

${ }^{10} \mathrm{~S}$. Wieder and E. Jaoudi, "Autobalancing radiometer," Am. J. Phys. 46(9), 935-937 (1978).
${ }^{11}$ J. Fendley and M. Hutchins, "Selective-surface solar radiometer," Am. J. Phys. 52(6), 550-555 (1984).

${ }^{12}$ F. Kissner, "Inexpensive photovoltaic radiometer," Am. J. Phys. 49(5), 439-442 (1981).

${ }^{13}$ U. Ganiel and O. Kedem, "Solar energy: How much do we receive?," Phys. Teach. 21, 573-575 (1983).

${ }^{14}$ M. P. Silverman and C. R. Silverman, "Cool in the kitchen: Radiation, conduction, and the Newton 'Hot Block,' experiment," Phys. Teach. 38, 82 (2000).

${ }^{15}$ D. R. Pitts and L. E. Sissom, Heat Transfer (McGraw Hill, New York, 1970).

${ }^{16}$ American Institute of Physics Handbook, edited by D. E. Gray (McGraw Hill, New York, 1966) and 〈http://en.wikipedia.org/wiki/Beer-Lambert-law〉.

${ }^{17}$ Multipurpose Lab Interface (MPLI), Vernier, 〈www.vernier.com〉.

${ }^{18}$ The altitude and azimuth of the Sun can be obtained for a specific day at $\langle$ http://aa.usno.navy.mil/data/docs/AltAz.html .

${ }^{19}$ Edmund Scientific offers a bag of broken cells for less than $\$ 10$, $\langle$ http://scientificsonline.com/〉. Preassembled solar cell detectors can be obtained from $\langle$ http://www.tandar.cnea.gov.ar/grupos/solar/sol-hpg.html $\rangle$.

${ }^{20}$ J. N. Bahcall, M. H. Pinsonneault, and S. Basu, "Solar models: Current epoch and time dependences, neutrinos, and helioseismological properties," Astrophys. J. 555, 990-1012 (2001).

${ }^{21} \mathrm{C}$. H. Hayn and V. Galvin, Jr., "Experimental determination of the mass of air molecules from the law of atmospheres," Am. J. Phys. 47, 69-70 (1979).

${ }^{22}$ A. Sánchez-Lavega, S. Pérez-Hoyos, and R. Hueso, "Clouds in planetary atmospheres: A useful application of the Clausius-Clapeyron equation," Am. J. Phys. 72, 767-774 (2004).

${ }^{23}$ F. Kasten and A. T. Young, "Revised optical air mass tables and approximation formula," Appl. Opt. 28, 4735-4738 (1989). 